\title{
Adipose Tissue-Residing Progenitors (Adipocyte Lineage Progenitors and Adipose-Derived Stem Cells (ADSC))
}

\author{
Ryan Berry $^{1} \cdot$ Matthew S. Rodeheffer ${ }^{2} \cdot$ Clifford J. Rosen $^{3} \cdot$ Mark C. Horowitz $^{1}$
}

Published online: 12 July 2015

(C) Springer International Publishing AG 2015

\begin{abstract}
The formation of brown, white, and beige adipocytes has been a subject of intense scientific interest in recent years due to the growing obesity epidemic in the USA and around the world. This interest has led to the identification and characterization of specific tissue-resident progenitor cells that give rise to each adipocyte population in vivo. However, much still remains to be discovered about each progenitor population in terms of their "niche" within each tissue and how they are regulated at the cellular and molecular level during healthy and diseased states. While our knowledge of brown, white, and beige adipose tissue is rapidly increasing, little is still known about marrow adipose tissue and its progenitor despite recent studies demonstrating possible roles for marrow adipose tissue in regulating the hematopoietic space and systemic metabolism at large. This chapter focuses on our
\end{abstract}

This article is part of the Topical Collection on Molecular Biology of Adult Stem Cells

Mark C. Horowitz

mark.horowitz@yale.edu

Ryan Berry

ryan.berry@yale.edu

Matthew S. Rodeheffer

Matthew.rodeheffer@yale.edu

Clifford J. Rosen

cjrofen@gmail.com

1 Department of Orthopaedics and Rehabilitation, Yale School of Medicine, 330 Cedar Street, New Haven, CT 06510, USA

2 Department of Molecular, Cell and Developmental Biology, Yale University and the Section of Comparative Medicine, Yale School of Medicine, 375 Congress Avenue, New Haven, CT 06510, USA

3 The Center for Clinical and Translational Research, Maine Medical Center Research Institute, Scarborough, ME 04074, USA current knowledge of brown, white, beige, and marrow adipose tissue with a specific focus on the formation of each tissue from tissue-resident progenitor cells.

Keywords Adipocytes · Adipocyte progenitors . Differentiation $\cdot$ White fat $\cdot$ Brown fat $\cdot$ Marrow fat

\section{Introduction}

Adipose tissues are heterogeneous organs distributed throughout the body that have important endocrine and metabolic roles. Due to the their functions in storing (white adipose tissue (WAT)) and burning calories (brown adipose tissue (BAT) and beige adipocytes) in the form of lipids, significant research has focused on understanding the mechanisms controlling WAT, BAT, and beige adipocyte formation. In recent years, significant advances in our knowledge about the cellular and developmental ontogeny of adipose tissue have shown that each of these three tissues are derived from developmentally distinct lineages and progenitor cells. This knowledge is now allowing for directed studies on understanding the molecular mechanisms regulating the formation and function of each tissue in vivo, with hopes for identifying targeted therapies for obesity and other metabolic diseases.

While our knowledge of WAT, BAT, and beige adipocyte has grown significantly in recent years, our knowledge of the development and metabolic significance of marrow adipose tissue (MAT) is comparatively primitive. However, recent and ongoing studies point to a distinct ontogeny and genetic signature of MAT. Additionally, MAT is becoming more and more appreciated as an endocrine organ with the ability to affect systemic metabolism, highlighting the importance of understanding the role of this often neglected adipose tissue in metabolic disease. In this chapter, we will discuss the 
unique features of WAT, BAT, MAT, and beige adipocytes with specific focus on their development from distinct tissue-resident progenitor cells.

\section{White Adipose Tissue}

White adipose tissue serves as both the body's major lipid reservoir and as an important endocrine organ that regulates systemic metabolism [1]. As an organ, WAT is relatively unique in that it is dispersed throughout the body in several distinct depots. WAT depots can be primarily separated into subcutaneous (SWAT) depots residing directly beneath the skin and visceral (VWAT) depots located within the abdominal cavity surrounding internal organs [2]. Regardless of location, each WAT depot is composed of a heterogeneous mixture of cells including immune, endothelial, and stromal populations as well as adipocyte lineage cells [3].

Within the adipocyte lineage, mature white adipocytes are terminally differentiated postmitotic cells that comprise the majority of WAT volume. The endocrine and metabolic functions of WAT are primarily carried out by white adipocytes, which store lipids in a large unilocular lipid droplet that fills the majority of the cell. In times of caloric deficit, white adipocytes catabolize stored lipids and release triglycerides into the circulation as an energy source for other tissues [4]. Additionally, white adipocytes secrete a number of hormones including adiponectin, resistin, leptin, TNF $\alpha$, and IL-6, which affect insulin sensitivity, inflammation, and feeding [1, 5]. Because of the central role of white adipocytes in regulating several metabolic processes and the marked expansion of WAT in obesity, regulation of adipocyte function and formation are potential therapeutic targets for obesity, type II diabetes mellitus, and cardiovascular disease.

The formation of mature adipocytes is accomplished at the cellular level through the differentiation of upstream adipocyte lineage cells, termed adipocyte precursors. Adipocyte precursors have been characterized in mice as tissue-resident cell populations with either small or large in vivo adipogenic capacity. Early adipocyte progenitors (APs) are characterized by the cell surface phenotype CD45-: CD31- (Lin-): CD29+: CD34+: Sca-1+: PdgfR $\alpha+$ : CD24+, and have high adipogenic capacity as demonstrated by their ability to generate an entire functional WAT depot following in vivo transplantation [6•]. Pre-adipocytes are an intermediate population of adipocyte precursors generated from the differentiation of APs [7•]. Pre-adipocytes share the same cell surface phenotype as APs, with the exception of being negative for expression of CD24 [7•, 8, 9]. Whereas APs have high adipogenic capacity, pre-adipocytes have much lower adipogenic capacity as demonstrated by the formation of only small numbers of adipocytes following in vivo transplantation $[7 \cdot, 8]$. In adults, the abundance of each precursor population is inversely correlated to their adipogenic capacity as APs are a rare cell population that comprise less than $0.1 \%$ of all stromal vascular fraction (SVF) cells (the heterogeneous mixture of all nonmature adipocyte cells within WAT) within WAT depots, while pre-adipocytes represent between 15 and $35 \%$ of WAT SVF cells by flow cytometry $[6 \bullet, 7 \bullet, 8]$. During early development in mice, connective tissue at the site of WAT formation is filled with $\mathrm{CD} 24+\mathrm{APs}$ that eventually give rise in situ to both CD24- pre-adipocytes and mature adipocytes $[7 \cdot, 10]$. While adipocyte precursors with in vivo adipogenic capacity have yet to be identified in humans, several similarities exist between murine and human WAT development.

In both mouse and human, white adipose tissue (WAT) forms early in life. In humans, lipid-filled subcutaneous white adipocytes first arise during the second trimester [11] with developed SWAT depots being present at birth [11-13]. In mice, lipid filled inguinal SWAT adipocytes are observed concomitantly with birth and a distinguishable SWAT depot develops within $24 \mathrm{~h}$ [10]. Murine VWAT develops shortly after murine SWAT as lipid-filled visceral white adipocytes become present on postnatal day 7 [14]. Less is known about human VWAT development, except that some human VWAT does not develop until after birth and there is little VWAT in nonobese humans at puberty [15-17]. Therefore in both species, SWAT forms at or prior to birth and VWAT forms shortly after birth.

The origin of WAT and adipocyte precursors has been a subject of debate for several years. Since the early 1900s when adipocytes were first observed within condensations of loose connective tissue during early development, WAT was assumed to be derived from a mesenchymal lineage [11]. However, publications over the last decade have proposed endothelial, hematopoietic, and pericyte origins for adipocyte lineage cells $[9,18-20]$. While multiple lineages have been proposed, more recent lineage tracing of adipocyte lineage cells using the double fluorescent $\mathrm{mT} / \mathrm{mG}$ reporter mouse line demonstrated that white adipocytes and adipocyte precursors are not of endothelial or hematopoietic origin, but are traced by expression of the primarily mesenchymal marker $\operatorname{PdgfR} \alpha$ in adipocyte progenitors [7•]. Inconclusive results have been published about the in situ location or "niche" of adipocyte progenitors. Pericytes are exclusively perivascular cells, and much of the hypothesis of the pericyte origin of adipocytes is based on observations that cells with markers of APs can be found along the vasculature [9]. Despite the high vascularity of WAT, it has also been reported that only a fraction of cells with markers of APs are found in close proximity to blood vessels [7•]. Therefore, the ontogeny of WAT and the AP niche are still a matter of some debate. As no exclusive marker of APs has yet been identified, it is possible that the currently recognized population of CD24+ APs is a heterogeneous population and that the markers currently available are therefore not specific enough to identify the true AP niche. 


\section{Brown and Beige Adipose Tissue}

Brown adipose tissue (BAT), like WAT, is found in discrete depots. However, the number and size of BAT depots is less than that of WAT depots. BAT develops during embryonic differentiation, before other adipose tissue depots. In mice, the majority of BAT resides in a single depot in the interscapular space (interscapular, axillary, and cervical pads) surrounded by back muscles. Because BAT develops early in life, it is most prominent in human newborns and was thought to disappear by adulthood. The pronounced presence in newborns is related to its major function of heat production, which is central for survival of small mammals, especially in the cold. Brown adipocytes in BAT contain large numbers of mitochondria that contain uncoupling protein-1 (UCP1). UCP1 disrupts the electrochemical proton gradient that drives ATP synthesis during oxidative phosphorylation by allowing the transport of protons across the inner mitochondrial membrane without passage through ATP synthase. The result is the burning of substrates (primarily stored triglycerides) to generate heat with little ATP production. The heat generated by BAT is distributed throughout the body via the circulation. The interscapular space contains large numbers of blood vessels to facilitate this heat transfer. It was thought for many years that BAT was not present in adult humans, or if present, there was too little to affect overall metabolism. Using more sophisticated PET/CT imaging, this perception changed a few years ago when UCP1-expressing adipocytes were discovered in the supraclavicular region in adults and positive uptake of FDG was noted during scanning in that same region [21, 22•, 23].

The majority of brown adipocytes arise from progenitor cells in the developing embryonic mesoderm. Using in vivo lineage tracing, brown adipocytes develop from skeletal muscle precursors that express Myf5, Pax7, and Engrailed [24•, 25, 26]. This is important because until these studies, Myf5 and $\operatorname{Pax} 7$ were thought to be expressed only in the myocyte lineage. Furthermore, PRDM16 (PRD-BF1-RIZ1 homologous domain containing 16) is a zinc finger containing transcription factor that regulates the cell fate between brown adipocytes and skeletal myocytes in vitro and is at least required for the maintenance BAT in vivo [24•, 27, 28]. Knockdown of PRDM16 using shRNA in brown adipocyte precursors in vitro caused the selected loss of genes expressed in brown fat (Ucp1, Cidea, Elov13). Reduction of PRDM16 in the same cells resulted in a marked increase in the expression of genes associated with myogenesis (Myod, myogenin, and myosin light chain, muscle creatine kinase) [24•]. Conversely, expression of PRDM16 in myoblasts resulted in their differentiation into brown adipocytes. Using a cre-lox system, PRDM16 was deleted in brown adipocyte progenitors and skeletal muscle in vivo [28]. While this conditional knockout did not affect the development of BAT, the size of the BAT depot increased as the mice aged. However, the increased size was not due to increased numbers of multilocular brown adipocytes, but rather the conversion of brown adipocytes to unilocular white adipocytes within the BAT depot (a phenomenon called "whitening"). This whitening correlated with a decrease in BAT-specific genes and an increase in WAT-specific genes [28]. The combination of in vitro and in vivo data suggests that PRDM16 regulates both the formation of brown adipocytes from early mesodermal $M y f 5$ expressing precursors and the maintenance of brown adipocyte function in adults, but that biological redundancy prevents PRDM16 from being necessary for the early fate switch in vivo [28].

Unlike BAT or WAT, beige adipocytes are not found in discrete depots but rather interspersed within WAT, especially in the subcutaneous inguinal depot. "Browning or beiging" of white adipose tissue is induced by cold exposure or other stimuli (i.e., $\beta$ adrenergic) that increase sympathetic tone [29]. Beige adipocytes are multilocular, contain large numbers of mitochondria, and express brown fat genes (i.e., Ucp1, $P g c 1 \alpha, C i d e a)$. PRDM16 is expressed in WAT depots and more in those that beige (i.e., inguinal) [30•]. Overexpression of PRDM16 in adipose tissue induces beige adipocyte differentiation in WAT and functions to block highfat diet-induced weight gain and improve glucose tolerance $[30 \bullet$.

The origin and development of beige adipocytes is less well described than that of brown or white adipocytes. Beige adipocytes and their progenitors do not trace with $M y f 5$, suggesting they come from a lineage distinct from brown fat [24•, 31]. To better define the origin of beige adipocytes, adipogenesis was followed using a newly developed mouse model that allows the permanent labeling of mature adipocytes with LacZ and is inducible (AdipoChaser) [32]. Browning of subcutaneous white fat in AdipoChaser mice by cold exposure shows that most beige adipocytes arise from a precursor population rather than from preexisting adipocytes [32]. Using limiting dilution analysis of the stromal vascular fraction of subcutaneous inguinal WAT, two kinds of adipocyte precursors were identified, white and beige [33•]. However, only the beige precursors assumed a thermogenic gene profile in response to $\beta$-adrenergic agonist. In addition, beige precursors could be separated from WAT precursors by expression of the cell surface proteins CD137 and transmembrane protein 26, which can be used to enrich for these precursors. These data suggest that beige and white adipocytes are distinct. However, this remains controversial. Others have reported that unilocular white adipocytes can transdifferentiate into beige adipocytes in response to cold or $\beta$-adrenergic agonist [29]. More recently, using a variety of reporter mice for lineage tracing and translating ribosomal affinity purification (TRAP) data suggest that Myh11 (a selective maker for smooth muscle-like cells) expressing cells differentiate into mature beige adipocytes, indicating the smooth muscle origin of beige but not brown adipocytes $[34,35]$. Taken together, these data suggest 
that both brown and beige adipocytes are important in regulating thermogenesis and whole animal metabolism. Both types of adipocytes share a set of genes associated with energy expenditure, although they also express unique genes, suggesting they are distinct. Because of their ability to influence whole body metabolism, they are primary targets to regulate obesity, glucose sensitivity, and cardiovascular disease.

\section{Bone Marrow Adipose Tissue}

Bone marrow (BM) is an extremely complex heterogeneous mixture of hematopoietic, immune, and skeletal lineage cells. Each cell lineage is represented within BM by cell populations that extend from true stem cells to mature functional effector cells. Early in life, the BM is "red" as it is predominantly occupied with hematopoietic cells. Development of BM adipocytes leads to "yellow" fatty marrow that replaces the "red" marrow within long bones of humans and mice at the time of peak bone acquisition and at other skeletal sites such as the spine later in life [36, 37]. Anecdotally, the yellow color of marrow adipose tissue (MAT) is distinct from white or brown adipose tissue especially in humans, suggesting it might be different than these other depots. One explanation for this difference may be the number of mitochondria in each type of adipocyte as white adipocytes have very few, brown adipocytes have many, and BM adipocytes have an intermediate number [38]. While BM adipocyte number increases as a result of normal aging, there are other known inducers of marrow adiposity. Commonly used drugs such as glucocorticoids and thiazolidinediones induce progressive marrow adiposity accompanied by rapid bone loss [39]. Mice fed for 8-12 weeks with a diet containing rosiglitazone, a potent PPAR $\gamma$ agonist that increases the sensitivity of cells to insulin, develop large numbers of BM adipocytes found extending through the metaphases into the diaphysis $[40 \bullet, 41]$. Rosiglitazone-induced adipogenesis is also associated with bone loss [41]. High-fat diet (HFD), irradiation, altered states of metabolism (e.g., calorie restriction and anorexia nervosa), gene mutations (e.g., loss of $E b f 1)$, strain differences, and estrogen deficiency all can lead to increased MAT.

Mesenchymal lineage cells including mesenchymal stem cells, stromal cells, osteoblasts, and adipocyte lineage cells also inhabit the BM [42•, 43]. Culture of mesenchymal stromal cells from whole BM resulted in the identification of fibroblast colony-forming units (CFU-F) that were selfrenewing and had the capacity to differentiate into all mesenchymal lineage cells [44]. Osterixl (Osxl) is a transcription factor required for osteoblast differentiation that is expressed immediately downstream of Runx2. Deletion of osx 1 results in an early arrest in osteoblast differentiation [45 ${ }^{\circ}$. Using lineage tracing in fluorescent reporter mice Osterix + cells have been shown to trace osteoblast lineage and BM stromal cells at different stages of mouse BM development [46•]. In a separate set of experiments, the majority of CFU-Fs ( $94 \%$ ) were traced with the leptin receptor (LepR) [47•]. These LepR+ cells also traced with Nestin, which is also found on CFU-Fs [47•]. Leptin+ cells traced most adipocytes and osteoblasts in adult BM. LepR + cells have also been reported to trace BM stromal cells that support hematopoietic stem cell maintenance [48]. Importantly, the LepR + traced BM population largely overlaps with Nestin-GFP+ population and the LepR+ BM cells $[46 \bullet, 47 \bullet, 49,50]$. In summary, these data suggest that mesenchymal progenitors are $\mathrm{Osx}+$ in neonatal $\mathrm{BM}$ and give rise to Nestin+ and LepR + cells in adult BM, which can differentiate down the adipocyte and osteoblast lineages. In a separate set of experiments, Gremlin-creER mice were crossed with a variety of fluorescent reporter mice to trace mesenchymal lineage differentiation [51•]. Gremlin was used because of its mesenchymal stem cell expression and because it is known to inhibit bone morphogenetic protein activity and be important in skeletal development $[52,53]$. Using lineage tracing with tamoxifen induction, Gremlin1-creER+ cells (Grem1+) never gave rise to Nes-GFP-expressing cells. However, osteoblasts, chondrocytes, myofibroblasts and BM stromal cells were all traced in Grem1-creER+ mice. Importantly, Grem 1+ cells failed to trace adipocytes in long bones and in the vertebra even after 1 year of induction [51•]. These data suggest that at least two separate progenitor populations are present in adult BM: the $O s x+, N^{+}+$, and LepR+ population, which give rise to osteoblasts, stromal cells, and adipocytes, and the Grem1+ population, which give rise to all of the mesenchymal lineage with the exception of adipocytes.

Although it is now accepted that BM adipocytes arise from mesenchymal stem cells, their ontogeny and relationship to white adipocytes, brown adipocytes, beige adipocytes, and osteoblasts remains only partially delineated. Because of the potential importance of MAT in local regulation of immune, skeletal, and hematopoietic events in the BM as well as the effect of MAT on whole body metabolism, we have started a detailed investigation of the lineage development of BM adipocytes.

In general, the amount of MAT is low in the long bones and vertebrae of young animals including humans and increases with age. In mice, strain has a significant effect on the amount of MAT. As an example, $\mathrm{C} 3 \mathrm{H} / \mathrm{HeJ}(\mathrm{C} 3 \mathrm{H})$ mice have very high endogenous levels of MAT even in young mice. In contrast, C57BL/6 (B6) mice have very low endogenous levels of MAT. Interestingly, $\mathrm{C} 3 \mathrm{H}$ mice have one of the highest endogenous bone densities (both trabecular and cortical) of any mouse strain examined. B6, on the other hand, have one of the lowest bone densities of inbred strains. These data are important because they contradict the dogma that high marrow fat correlates with low bone density. The effect of MAT on bone density in humans is also unclear. Obesity is correlated with increased MAT, and studies in older men, 
postmenopausal women, and children show that obesity increases the risk of fractures [54•]. However, there are currently no data demonstrating a causative relationship between MAT and fractures. To better understand the relationship of diet, MAT, and bone mineral density, we studied the effects of a high-fat diet (HFD) on bone mass and MAT in B6 mice following long- (84 days in males) or short-term (14 days in males and females) feeding. We found that consumption of a HFD rapidly increases the volume of MAT but has little or no effect on trabecular and cortical bone as measured by DXA, histomorphometry, and micro-CT [54•]. These data indicate that the amount of MAT does not always correlate with changes in bone.

Because of the generalized low endogenous level of MAT in most mice, study of marrow adipocytes requires induction of marrow adipogenesis. We induce MAT formation through feeding the mice a diet containing the PPAR $\gamma$ agonist rosiglitazone for a minimum of 6 weeks, lethally irradiating (700 rads) and reconstituting mice with syngeneic BM cells or a variety of other treatments. In non-treated mice, MAT can be observed above the growth plate, in the secondary center of ossification and just below the growth plate in the primary spongiosa. Treatment with rosiglitazone or irradiation results in a marked increase in MAT above the growth plate and extending from the growth plate through the metaphysis into the diaphysis filling the bone $[40 \bullet, 55]$. This filling of the medullary canal in both the tibia and femur is similar to that seen in humans by the time they reach 40 years old [56]. In addition to this regulated MAT (rMAT), which is not always present but forms in response to a stimulus, there is evidence for another type of MAT, which we refer to as constitutive MAT (cMAT). The characteristics of cMAT are that it is present in bone prior to the appearance of rMAT, it is anatomically located in the distal tibia and caudal vertebra, and it differs from rMAT by its lipid profile [57]. The relationship between and functional roles of cMAT and rMAT are unknown.

Despite the ease of inducing marrow adipogenesis, both the presence of bone, which makes accessing MAT difficult, and the heterogeneous mixture of cells within the BM, which makes lineage tracing difficult, have hindered the study of MAT. Therefore, new techniques have been developed to circumvent these issues and allow for the study of MAT in vivo. Our laboratory and others have utilized a variety of fluorescent reporter mice to trace the lineage of bone cells. To determine the origin of BM adipocytes, we have performed lineage tracing using the fluorescent $m T / m G$ reporter mouse in concert with various mouse models driving cre-recombinase from lineage-specific promoters. In $m T / m G$ mice, expression of cre-recombinase results in permanent removal of the membrane-targeted dTomato $(\mathrm{mT})$ cassette and expression of the membrane-targeted eGFP $(\mathrm{mG})$ cassette, resulting in "flipping" from dTomato + to eGFP+ cells. The $m T / m G$ model is extremely useful for lineage tracing because expression of cre-recombinase in progenitor cells results in the permanent expression of eGFP in both progenitor and daughter cells. Following the induction of marrow adipogenesis with either rosiglitazone diet or lethal irradiation with bone marrow reconstitution, femurs are collected and one of two approaches is used to visualize the in vivo fluorescence of the BM adipocytes. In the first, the femoral head is removed and a 20 -gauge needle is inserted through the medullary shaft until it protrudes out the distal end of the femur. The BM plug is gently blown onto a microscope slide using a 5-cc syringe, cover-slipped and the cells visualized by confocal microscopy (Fig. 1). A second approach involves fixing the dissected bones in formalin, decalcifying the bones for more than 2 weeks in EDTA, and processing the bones for frozen sections. Additionally, both these techniques allow for subsequent fluorescent labeling of intracellular lipid with the fluorescent lipophilic dye LipidTox, which allows for the rapid identification of adipocytes within the BM cells (Fig. 1).

Using this approach, lineage tracing shows that BM adipocytes are uniformly dTomato+ in Vav1-cre: $m T / m G$ mice, which traces hematopoietic stem cells and their progeny. This is important because it has been reported that WAT can arise from hematopoietic progenitor cells [58], although more recent data using the $m T / m G$ reporter mouse does not support this conclusion [7•]. To further characterize the BM adipocyte progenitor, $\mathrm{B} 6$ or $\mathrm{mT} / \mathrm{mG}$ mice were lethally irradiated (1000 rads) and reconstituted with $\mathrm{B} 6$ or $\mathrm{mT} / \mathrm{mG} \mathrm{BM}$ cells. Reconstitution of irradiated $\mathrm{mT} / \mathrm{mG}$ mice with B6 BM resulted in dTomato+ adipocytes, indicating that the BM adipocyte progenitor resides in the irradiated host and do not arise from transplanted cells. In contrast, reconstitution of irradiated B6 mice with $\mathrm{mT} / \mathrm{mG}$ BM resulted in adipocytes that were not dTomato + . These data indicate that $\mathrm{BM}$ adipocyte progenitors are radio-resistant, non-hematopoietic cells that reside in situ in long bones and do not come from the circulation.

Prdm16 and Ucp-1 expression from whole B6 tibia has been reported, suggesting the presence of preformed brown or beige adipocytes [38]. We have found that BM adipocytes are not lineage related to brown adipocytes as they are uniformly dTomato+ in Myf5-cre:mT/mG mice. Moreover, all BM adipocytes we have observed, whether constitutively present or induced, were unilocular. As mentioned previously, brown and beige adipocytes are multilocular. Placing B6 mice on a methionine-restricted (MR) diet extends life span, improves insulin sensitivity, reduces WAT, and reduces bone mass [59]. In contrast, MR induces a significant increase in MAT and concomitant "beige-ing" of inguinal WAT (J. Hens, G. Ables, M. Horowitz unpublished). However, BM adipocytes from MR mice show no "beige-ing" (G. Ables, J. Hens, M. Horowitz, unpublished). These data suggest that BM adipocytes do not derive from the brown or beige lineages.

It was reported recently that white adipocyte progenitors in vivo express $P d g f R \alpha$ resulting in tracing of all white 
Fig. 1 Induction and lineage tracing of MAT. a Adipocytes are largely absent from the bone marrow of C57BL/6 mice (left panel) unless bone marrow adipogenesis is induced with one of several techniques (right panel). $\mathbf{b}$ Bone marrow adipocytes express the adipocyte hormone adiponectin as shown by eGFP+ bone marrow adipocyte plasma membranes in Adiponectincre: $m T / m G$ mice fed a rosiglitazone diet for 8 weeks
A

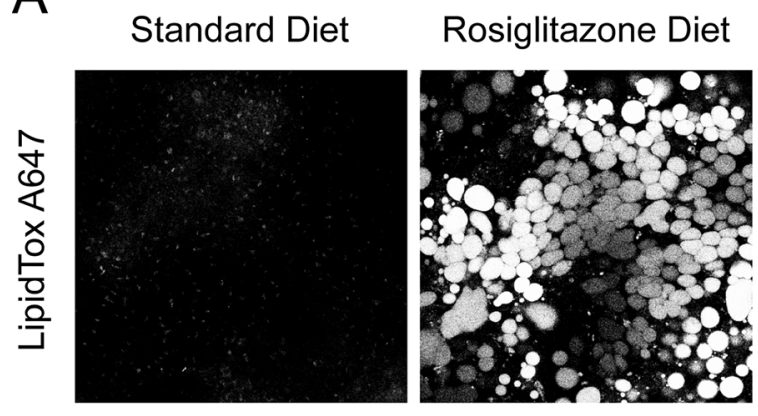

B
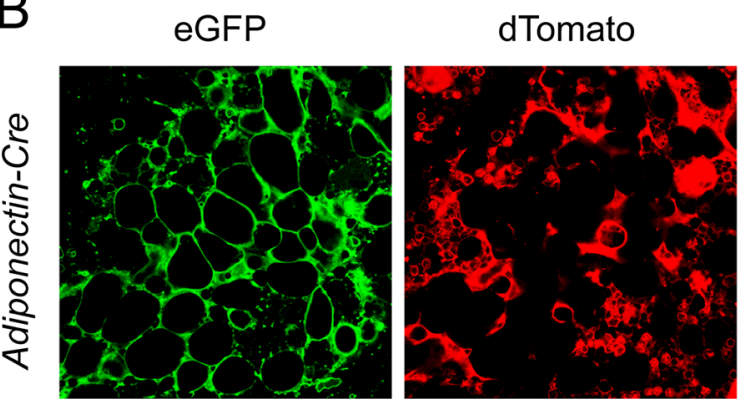

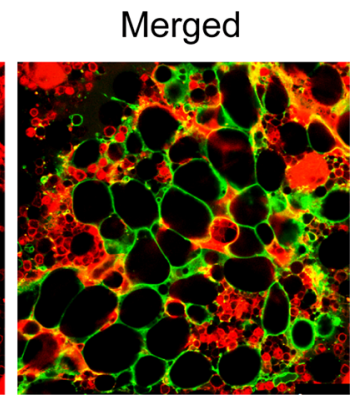

adipocytes in $P d g f R \alpha$-cre: $m T / m G$ mice [7•]. Despite the morphological similarities between white and bone marrow adipocytes, we have not observed similar uniform tracing of bone marrow adipocytes as only a subset (approx. $50 \%$ ) of BM adipocytes were e $\mathrm{GFP}^{+}$in $P d g f R \alpha$-cre: $m T / m G$ mice. As mentioned above, Osxl expression was believed to be osteoblast specific. However, Osx1+ BM cells trace a population of mesenchymal progenitor cells. In addition, $x$-irradiation of Leprcre/Tomato and Osx/Tomato reporter mice resulted in Tomato+, perilipin+, and BODIPY (lipid staining dye) adipocytes [46•]. Moreover, it was shown that BM adipocytes were traced by expression of $O s x-1[60,61]$. Importantly, we observe uniform dTomato ${ }^{+}$adipocytes and adipocyte progenitors in visceral WAT, subcutaneous WAT, and BAT depots in Osx-1-cre: $m T / m G$ mice. In contrast, all BM adipocytes are traced in irradiated or rosiglitazone treated $O s x-1$-cre: $m T / m G$ mice, while tracing a subset of cells on bone surfaces and osteocytes in this same model [62•]. These data suggest that tracing by osx-1 distinguishes $\mathrm{BM}$ adipocytes from other types of adipocytes.

\section{Conclusions and Perspectives}

Although fat cells have been studied for hundreds of years, interest in adipocyte biology has accelerated over the past 20 years and is the subject of renewed interest due to their role in the growing epidemic of obesity, cardiovascular disease, and the loss of metabolic regulation. For many years, it was widely accepted that there were two types of adipocytes, white and brown. White adipocytes were found in discrete depots subcutaneously or in the peritoneal cavity in close association with major organs. It has only been 7 years since the cell surface phenotype (Lin-, CD29+, CD34+, Sca1+, CD24+) of WAT progenitors was identified in vivo [6॰] and 2 years since the adipocyte cellular lineage was established in vivo [7॰].

In mice, brown adipocytes are found in a single interscapular depot and the cells disappear with age. In humans, brown adipocytes were observed to be most prominent in the young and, as in mice, disappear with age. However, recent studies show that brown adipocytes persist into adulthood. Substantial data now indicates that browning or beiging of WAT is the result of the differentiation of beige adipocyte progenitors present in WAT. This result, along with the identification of markers defining beige adipocytes, demonstrates that beige adipocytes differentiate from a lineage distinct from white and brown adipocytes. These cells play a role in thermogenesis and the regulation of obesity, at least in mice. The functional role of beige adipocytes in regulating metabolism in humans remains to be clearly demonstrated.

While much attention has been given to the paracrine, endocrine, and metabolic roles of white, brown, and beige adipocytes, the importance of bone marrow adipocytes has been marginalized if not outright ignored. Adipocytes were identified in the BM more than a century ago, but unlike adipose tissue in other depots, these cells have never been considered more than "filler" for other "more important" BM cells [63]. Although this concept is out-of-date, it persists today. MAT is generally low in young mice and humans and increases with age for reasons that are not well understood. Importantly, a variety of other stressors or agents that cause BM injury 
induce increased MAT. The accumulation of MAT has clinical implications. Young women with anorexia nervosa and mice on a calorie-restricted diet have increased MAT, which correlates with increased fracture rate [64•]. The trauma of bone fracture itself induces MAT [47•]. Interestingly, fracture repair is mediated, at least in part, by mesenchymal progenitor cells that are likely descendants of $\mathrm{Osx}+, \mathrm{LepR}+$, Nestin+, and Grem1+ stromal progenitor cells and are traced by alpha smooth muscle actin $[65,66]$. Additionally, multiple myeloma in the BM had been associated with increased MAT [67]. Thus, it is commonly perceived that the presence of MAT results is bone loss with biomechanically compromised bones. However, little if any data is currently available demonstrating a causal relationship between MAT and low bone volume. In fact, data showing B6 mice have low MAT and low bone mass while $\mathrm{C} 3 \mathrm{H}$ mice that have high MAT and high bone mass indicates that a causal relationship does not exist. The type of induction appears to be another fact in this relationship. Treatment with TZDs potently induces MAT and correlates with decreased bone mass due to high rates of bone loss from enhanced osteoclastic activity [41]. In contrast, high-fat diet feeding, which induces MAT does not result in loss of bone [54•]. Inflammation has been linked to the deleterious effects of visceral WAT [68 $]$. Therefore, one possible explanation for the bone loss is that MAT is involved with a localized inflammatory response with the release of cytokines that induce osteoclast activation [68•].

Based on morphological similarities between WAT and MAT, we initially reasoned that the MAT progenitor was identical to the WAT progenitor. However, we were unable to identify the WAT progenitor in B6 BM through flow cytometry. In subsequent experiments, we have shown that MAT does not express WAT or BAT markers and appears unable to beige in vivo. In addition, these cells express markers unique to MAT. Thus, the preponderance of the data suggests that MAT is distinct from other types of fat and likely arises from a different progenitor. Although BM adipocytes and osteoblasts arise from BM MSCs, their lineage development and relationship to each other is unclear. Lineage tracing shows that cells with markers of BM adipocytes are seen on bone surfaces [62•]. This suggests that cells intermediate in differentiation between MSCs and mature BM adipocytes are on the endosteum or trabecular bone surfaces, and is consistent with our data that BM adipocytes arise from a distinct progenitor.

In summary, these studies suggest that BM adipocytes have an important role in regulating bone function and may contribute to overall body homeostasis. However, much remains to be done to identify the origin (differentiation) and function (regulatory activity) of marrow adipocytes.

\section{Compliance with Ethics Guidelines}

Conflict of Interest Ryan Berry and Clifford J. Rosen and declare that they have no conflict of interest.

Matthew S. Rodeheffer reports grants from NIH, during the conduct of the study.

Mark C. Horowitz reports personal fees from Ember Pharmacuticals and personal fees and non-financial support from Orentreich Foundation for the Advancement of Science, outside the submitted work.

Human and Animal Rights and Informed Consent Among cited articles where one of the authors of the current report were authors, local Institutional Review Board approval was obtained and maintained for studies where human (or animal) subjects research was performed.

\section{References}

Papers of particular interest, published recently, have been highlighted as:

- Of importance

1. Galic S, Oakhill JS, Steinberg GR. Adipose tissue as an endocrine organ. Mol Cell Endocrinol. 2010;316:129-39.

2. Cinti S. The adipose organ. Prostaglandins Leukot Essent Fat Acids. 2005;73:9-15.

3. Cryer A, Van RLR. New perspectives in adipose tissue: structure, function and development. Butterworth-Heinemann, 2014.

4. Lass A, Zimmermann R, Oberer M, Zechner R. Lipolysis-a highly regulated multi-enzyme complex mediates the catabolism of cellular fat stores. Prog Lipid Res. 2011;50:14-27.

5. Kershaw EE, Flier JS. Adipose tissue as an endocrine organ. J Clin Endocrinol Metab. 2004;89:2548-56.

6. Rodeheffer MS, Birsoy K, Friedman JM. Identification of white adipocyte progenitor cells in vivo. Cell. 2008;135:240-9. This paper was the first to identify in vivo adipocyte progenitor cells as tissue resident cells with the cell surface phenotype of Lin-; CD29+; CD34+; Sca-1+; CD24+. This progenitor population was shown to be capable of reconstituting a functional WAT depot upon transplantation into lipodystrophic mice.

7. Berry R, Rodeheffer MS. Characterization of the adipocyte cellular lineage in vivo. Nat Cell Biol. 2013;15:302-8. This paper demonstrated the ontogeny of white adipocytes through lineage tracing. Additionally, a distinct population of pre-adipocyte cells were identified as being derived from adipocyte progenitor cells in vivo. PdgfR $\alpha$ was demonstrated to be a marker of adipocyte progenitors and pre-adipocytes.

8. Lee YH, Petkova AP, Mottillo EP, Granneman JG. In vivo identification of bipotential adipocyte progenitors recruited by $\beta 3$ adrenoceptor activation and high-fat feeding. Cell Metab. 2012;15:480-91.

9. Tang W, Zeve D, Suh JM, Bosnakovski D, Kyba M, Hammer RE, et al. White fat progenitor cells reside in the adipose vasculature. Science. 2008;322:583-6.

10. Birsoy K, Berry R, Wang T, Ceyan O, Tavazoie S, Friedman JM. Analysis of gene networks in white adipose tissue development reveals a role for ETS2 in adipogenesis. Development. 2011;138: 4709-19.

11. Poissonnet CM, Burdi AR, Bookstein FL. Growth and development of human adipose tissue during early gestation. Early Hum Dev. 1983;8:1-11. 
12. Poissonnet CM, Burdi AR, Garn SM. The chronology of adipose tissue appearance and distribution in the human fetus. Early Hum Dev. 1994;10:1-11.

13. Berg W. The development of human fat. Z Morph Anthrop. $1911 ; 13$.

14. Han J, Lee JE, Jin J, Lim JS, Oh N, Kim K, et al. The spatiotemporal development of adipose tissue. Development. 2011;138: 5027-37.

15. Fox K, Peters D, Armstrong N, Sharpe P, Bell M. Abdominal fat deposition in 11-year-old children. Int J Obes Relat Metab Disord. 1993;17:11-6.

16. Siegel MJ, Hildebolt CF, Bae KT, Hong C, White NH. Total and intraabdominal fat distribution in preadolescents and adolescents: measurement with MR imaging. Radiology. 2007;242:846-56.

17. Wassermann F. On the formation of appendices epiploicae in man, with consideration of the development of the fat organs therein. Ergeb Anat Anz. 1927;63.

18. Tran KV, Gealekman O, Frontini A, Zingaretti MC, Morroni M, Giordano A, et al. The vascular endothelium of the adipose tissue gives rise to both white and brown fat cells. Cell Metab. 2012;15: 222-9.

19. Crossno Jr JT, Majka SM, Grazia T, Gill RG, Klemm DJ. Rosiglitazone promotes development of a novel adipocyte population from bone marrow-derived circulating progenitor cells. J Clin Invest. 2006;116:3220-8.

20. Majka SM, Miller HL, Sullivan T, Erickson PF, Kong R, WeiserEvans M, et al. Adipose lineage specification of bone marrowderived myeloid cells. Adipocyte. 2012;1:215-29.

21. Cypess AM, Lehman S, Williams G, Tal I, Rodman D, Goldfine $\mathrm{AB}$, et al. Identification and importance of brown adipose tissue in adult humans. N Engl J Med. 2009;360:1509-17.

22. Nedergaard J, Gengtsson T, Cannon B. Unexpected evidence for active brown adipose tissue in adult humans. Am J Physiol Endocrinol Metab. 2007;293:E444-52. This review discusses the identification and physiological significance of active brown adipose tissue in humans that has been observed through FDG PET scans. The authors conclude from the published studies that variation in brown adipose tissue plays a role in human metabolism and is therefore a pharmacological target for obesity.

23. Saito M, Okamatsu-Oqura Y, Matsushita M, Watanabe K, Yonesiro T, Nio-Kobayashi J, et al. High incidence of metabolically active brown adipose tissue in healthy adult humans: effects of cold exposure and adiposity. Diabetes. 2009;58:1526-31.

24. Seale O, Bjork B, Yang W, Kajimura S, Chin S, Kung S, et al. PRDM16 controls a brown fat/skeletal muscle switch. Nature. 2008;454:961-7. This paper demonstrates a distinct ontogeny for brown adipose tissue and white adipose tissue by demonstrating through lineage tracing that brown adipose tissue arises from precursor cells that express the myogenic transcription factor Myf5. The authors conclude from in vitro knockdown and overexpression studies as well as in vivo knock-out that PRDM16 drives the differentiation of brown adipocytes from a precursor cell expressing myoblast markers.

25. Lepper C, Fan CM. Inducible lineage tracing of Pax7-descendant cells reveals embryonic origin of adult satellite cells. Genesis. 2010;48:424-36

26. Atit R, Sgaier SK, Mohamed OA, Taketo MM, Dufort D, Joyner AL. Beta-catenin activation is necessary and sufficient to specify the dorsal dermal fate in the mouse. Dev Biol. 2006;296:164-76.

27. Seale P, Kajimura S, Yang W, Chin S, Rohas LM, Uldry M, et al. Transcriptional control of brown fat determination by PRDM16. Cell Metab. 2007;6:38-54.

28. Harms MJ, Ishibashi J, Wang W, Lim HW, Govama S, Sato T, et al. Prdm16 is required for the maintenance of brown adipocyte identity and function in adult mice. Cell Metab. 2014;19:593-604
29. Vitali A, Muran I, Zingaretti MC, Frontini A, Ricquier D, Cinti S. The adipose organ of obesity-prone $\mathrm{C} 57 \mathrm{BL} / 6 \mathrm{~J}$ mice is composed of mixed white and brown adipocytes. J Lipid Res. 2012;53:619-29.

30. Seale P, Conroe HM, Estall J, Kajimura S, Frontini A, Ishibashi J, et al. Prdm16 determines the thermogenic program of subcutaneous white adipose tissue in mice. J Clin Invest. 2011;121:96-105. This paper demonstrated that the brown determination factor PRDM16 is expressed in subcutaneous but not other white adipose tissues. This was a significant finding as subcutaneous white adipose tissue can "beige" in response to various stimuli.

31. Sanchez-Gurmaches J, Hung CM, Sparks CA, Tang Y, Li H, Guertin DA. PTEN loss in the Myf5 lineage redistributes body fat and reveals subsets of white adipocytes that arise from Myf5 precursors. Cell Metab. 2012;16:348-62.

32. Wang QA, Tao C, Gupta RK, Scherer PE. Tracking adipogenesis during white adipose tissue development, expansion and regeneration. Nat Med. 2013;19:1338-44.

33. Wu J, Bostr m P, Sparks LM, Ye L, Choi JH, Giang AH, et al. Beige adipocytes are a distinct type of thermogenic fat cell in mouse and human. Cell. 2012;150:366-76. This paper identifies beige adipocytes as a population of adipocytes that are distinct at the molecular level from white and brown adipocytes and are capable of significant $U C P 1$ expression in response to stimuli such as the polypeptide irisin.

34. Miano JM, Cserjesi P, Ligon KL, Periasamy M, Olson EN. Smooth muscle myosin heavy chain exclusively marks the smooth muscle lineage during mouse embryogenesis. Circ Res. 1994;75:803-12.

35. Long JZ, Svensson KJ, Tsai L, Zeng X, Roh HC, Kong X, et al. A smooth muscle-like origin for beige adipocytes. Cell Metab. 2014;19:810-20.

36. Moerman EJ, Teng K, Lipschitz DA, Lecka-Czernik B. Aging activates adipogenic and suppresses osteogenic programs in mesenchymal marrow stroma/stem cells: the role of PPAR- $\gamma 2$ transcription factor and TGF- $\beta$ /BMP signaling pathway. Aging Cell. 2004;3:379-89.

37. Moore SG, Dawson KL. Red and yellow marrow in the femur: agerelated changes in appearance at MR imaging. Radiology. 1990;175:219-23.

38. Krings A, Rahman S, Huang S, Lu Y, Czernik PJ, LeckaCzernik B. Bone marrow fat has brown adipose tissue characteristics, which are attenuated with aging and diabetes. Bone. 2012;50:548-52.

39. Lecka-Czernik G, Moerman EJ, Grant DF, Lehmann JM, Manolagas SC, Jilka RL. Divergent effects of selective peroxisome proliferator-activated receptor-gamma 2 ligands on adipocyte versus osteoblast differentiation. Endocrinology. 2002;143:2376-84.

40. Horowitz M, Berry R, Fretz J, Nelson T, Church C, Doucette C, et al. Bone marrow adipogenesis. J Bone Miner Res. 2013;28:S17. This abstract described the ability to study the formation of adipocytes within the bone marrow at the cellular and molecular level following induction of bone marrow adipogenesis through two techniques: feeding of rosiglitazone-enriched diet and irradiation/ bone marrow reconstitution. These findings allow for future studies of the metabolic impact of bone marrow adipogenesis in vivo.

41. Ackert-Bicknell CL, Shockley KR, Horton LG, Lecka-Czernik B, Churchill GA, Rosen CJ. Strain-specific effects of rosiglitazone on bone mass, body composition, and serum insulin-like growth factor-I. Endocrinology. 2009;150:1330-40.

42. Bianco P, Cao X, Frenette PS, Mao JJ, Robey P, Simmons PJ, et al. The meaning, the sense and the significance: translating the science of mesenchymal stem cells into medicine. Nat Med. 2013;19:3542. This review discusses our current knowledge of mesenchymal stem cells (MSCs) with an emphasis on their identity, location, functional ability and limitatons that have limited their therapeutic use thus far. 
43. Caplan AI. Mesenchymal stem cells. J Orthop Res. 1991;9:641-50.

44. Friedenstein JA, Petrakova KV, Kurolesova AI, Frolova GP. Heterotopic of bone marrow. Analysis of precursor cells for osteogenic and hematopoietic tissues. Transplantation. 1968;6:234-47.

45. Nakashima K, Zhou X, Kunkel G, et al. The novel zinc fingercontaining transcription factor osterix is required for osteoblast differentiation and bone formation. Cell. 2002;108:17-29. This paper identified Osterix (Osx) as a transcription factor expressed downstream of Runx2 in mesenchymal cells within the bone. As Osx-null mice were found to not develop bone as the mesenchymal cells within bone failed to deposit bone matrix, Osx was determined to be a transcription factor neccessary for osteoblast differentiation.

46. Mizoguchi T, Pinho S, Ahmed J, Kunisaki Y, Hanoun M, Mendelson A, et al. Osterix marks distinct waves of primitive and definitive stromal progenitors during bone marrow development. Dev Cell. 2014;29:340-9. This paper provides novel insights into the contribution of osterix (Osx) expressing cells to the formation of skeletal and stromal cells within the bone. Although Osx has been thought as a marker of commited osteoblasts, the authors find that Osx expressing cells give rise to transient and long lived stomal populations as well as osteolineage cells at different times during development.

47. Zhou BO, Yue R, Murphy MM, Peyer JG, Morrison SJ. Leptinreceptor-expressing mesenchymal stromal cells represent the main source of bone formed by adult bone marrow. CT Stem Cell. 2014;15:154-68. This paper demonstrates that the Leptin Receptor (LepR) is a marker of quiescent cells within the bone marrow that can proliferate and generate new bone, cartilage and adipocytes in adult animals. The authors show that LepR cells arise postnatally and function as adult mesenchymal stromal cells in vivo.

48. Ding L, Morrison SJ. Haematopoietic stem cells and early lymphoid progenitors occupy distinct bone marrow niches. Nature. 2013;495:231-5.

49. Pinho S, Lacombe J, Hanoun M, Mizoguchi T, Bruns I, Kunisaki Y, et al. PDGFR $\alpha$ and CD51 mark human Nestin+ sphere-forming mesenchymal stem cells capable of hematopoietic progenitor cell expansion. J Exp Med. 2013;210:1351-67.

50. Chan CKF, Seo EY, Chen JY, Lo D, McArdle A, Sinha R, et al. Identification and specification of mouse skeletal stem cell. Cell. 2015;160:285-98.

51. Worthley DL, Churchill M, Compton JT, Tailor Y, Rao M, Si Y, et al. Gremlin 1 identifies a skeletal stem cell with bone, cartilage, and reticular stromal potential. Cell. 2015;160:269-84. This paper demonstrates that Gremlin 1 is a marker of multi-potent cells within the bone marrow capable of giving rise to bone, cartilage and stromal cells but not adipocytes in vivo. This marker, in addition to LepR and Nestin, mark a population of cells capable of repairing the postnatal skeleton.

52. Hsu DR, Economides AN, Wang X, Eimon PM, Harland RM. The Xenopus dorsalizing factor Gremlin identifies a novel family of secreted proteins that antagonize BMP activities. Mol Cell. 1998;1:673-83.

53. Canalis E, Parker K, Zanotti S. Gremlin1 is required for skeletal development and postnatal skeletal homeostasis. J Cell Physiol. 2012;227:269-77.

54. Doucette CR, Horowitz MC, Berry R, MacDougald OA, Anunciado-Koza R, Koza RA, et al. A high fat diet increases bone marrow adipose tissue (MAT) but does not alter trabecular or cortical bone mass in C57BL/6J mice. Cell Physiol. 2015;230:2032-7. This paper addresses the link between obesity and increased prevalence of fractures by studying the impact of bone marrow adipocytes on bone remodeling. The authors find that a highfat diet capable of inducing both obesity and bone marrow adipogenesis does not result in significant changes in bone volume that could explain the observed increased fracture rates.

55. Bryon PA, Gentilhomme O, Fiere D. Histomorphometric analysis of bone-marrow adipose density and heterogeneity in myeloid aplasia and dysplasia. Pathol Biol (Paris). 1979;27:209-13.

56. Moore SG, Dawson KL. Red and yellow marrow in the femur: agerelated changes in appearance at MR imaging. Radiology. 1990;175:219-23.

57. Scheller EL, Rosen CJ. What's the matter with MAT? Marrow adipose tissue, metabolism, and skeletal health. Ann N Y Acad Sci. 2014;1311:14-30.

58. Majka SM, Fox KE, Psilas JC, et al. De novo generation of white adipocytes from the myeloid lineage via mesenchymal intermediates is age, adipose depot, and gender specific. Proc Natl Acad Sci U S A. 2010;107:14781-6.

59. Ables GP, Perrone CE, Orentreich D, Orentreich N. Methioninerestricted $\mathrm{C} 57 \mathrm{BL} / 6 \mathrm{~J}$ mice are resistant to diet-induced obesity and insulin resistance but have low bone density. PLoS ONE. 2012;7: e51357.

60. Liu Y, Strecker S, Wang L, Kronenberg MS, Wang W, Rowe DW, et al. Osterix-cre labeled progenitor cells contribute to the formation and maintenance of the bone marrow stroma. PLoS ONE. 2013;8: e71918.

61. Chen J, Shi Y, Regan J, Karuppaiah K, Ornitz DM, Long F. OsxCre targets multiple cell types besides osteoblast lineage in postnatal mice. PLoS ONE. 2014;9:E85161.

62. Horowitz M, Berry R, Webb R, Nelson T, Xi Y, Doucette CR, et al. Bone marrow adipocytes are distinct from white or brown adipocytes. J Bone Miner Res. 2014;29:S62. This abstract describes recent findings of the ontogeny and morphology of bone marrow adipocytes depicting bone marrow adipocytes as a distinct adipocyte population. As bone marrow adipocytes are derived from Osx1+; Myf5- precursor cells; brown adipocytes are derived from $\mathrm{Myf5}+$ precursor cells; and white adipocytes are not derived from Osx1+ precursor cells; bone marrow adipocytes have a distinct ontogeny.

63. Gimble JM, Robinson CE, Wu X, Kelly KA. The function of adipocytes in the bone marrow stroma: an update. Bone. 1996;19:421-8.

64. Fazeli PK, Horowitz MC, MacDougald OA, Scheller EL, Rodeheffer MS, Rosen CJ, et al. Marrow fat and bone-new perspectives. J Clin Endocrinol Metab. 2013;98:935-45. This review discusses recent studies uncovering the functional significance of bone marrow adipose tissue in mouse and human. The cumulative data suggests that in most circumstances, their is an inverse relationship between marrow adipose tissue and bone density/bone strength.

65. Torreggiani E, Matthews BG, Pejda S, Matic I, Horowitz MC, Grcevic D, et al. Preosteocytes/osteocytes have the potential to dedifferentiate becoming a source of osteoblasts. PLoS ONE. 2013;6:e75204.

66. Matthews BG, Grcevic D, Wang L, Hagiwara Y, Roguljic H, Joshi $\mathrm{P}$, et al. Analysis of $\alpha$ SMA-labeled progenitor cell commitment identifies notch signaling as an important pathway in fracture healing. J Bone Miner Res. 2014;29:1283-94.

67. Reagan MR, Liaw L, Rosen CJ, Ghobrial IR. Dynamic interplay between bone and multiple myeloma: emerging roles of the osteoblast. Bone. 2015;75:161-9.

68. Tilg H, Moschen AR. Adipocytokines: mediators linking adipose tissue, inflammation and immunity. Nat Rev Immunol. 2006;6: 772-83. This review describes our current knowledge of cytokines expressed and secreted from mature adipocytes. As these cytokines or adipokines are known to influence metabolism, inflammation and immunity, this review describes the many important endocrine and paracrine functions of adipose tissue. 